\title{
The Fairness of Solidarity Bills under the Solidarity Value of Nowak and Radzik
}

\author{
Lawrence Diffo Lambo ${ }^{1}$ and Pierre Wambo ${ }^{2}$ \\ ${ }^{1}$ Department of Mathematics, Ecole Normale Supérieure, Yaoundé, Cameroon \\ ${ }^{2}$ Department of Mathematics, Ecole Normale Supérieure, Maroua, Cameroon \\ Correspondence should be addressed to Lawrence Diffo Lambo; yldiffo@yahoo.fr
}

Received 8 December 2014; Revised 26 March 2015; Accepted 1 April 2015

Academic Editor: Walter Briec

Copyright (C) 2015 L. Diffo Lambo and P. Wambo. This is an open access article distributed under the Creative Commons Attribution License, which permits unrestricted use, distribution, and reproduction in any medium, provided the original work is properly cited.

\begin{abstract}
The solidarity value is a variant of the well-known Shapley value in which some sense of solidarity between the players is implemented allowing the disabled to receive help from the fortunate ones. We investigate on how fairly solidarity expenses are shared. We discuss the unwanted side effect of someone paying undue solidarity contributions as far as reversing his condition from a privileged to a needy person. A deeper case study is conducted for two classes of TU games that we obtain by modeling two real world business contexts. Here, we trace all player to player transfers of funds that arise when solidarity actions are processed, and we answer the question of who settles the solidarity bills. Also, we obtain the threshold position of a player below which he gets solidarity help, but above which he instead pays out donation.
\end{abstract}

\section{Introduction}

This paper is on the solidarity value of Nowak and Radzik (1994) [1]. A TU game models a business environment where it is of public awareness that any coalition of players $S$ would produce a known quantity of wealth $v(S)$ if all its members join hands as partners and act in agreement. Each player then receives his individual share from a side distribution of $v(S)$. A player in $S$ is free to either accept an agreement in $S$ or to quit $S$ and seek different opportunities with other coalitions. Which coalitions will rationally be formed? And which individual shares are likely to be agreed on in the side distribution? These two interwoven questions are the basic challenge to scientific research in TU games. The Shapley value (Shapley, 1953) [2] is a kind of social planner's idea assuming that the unitarian grand coalition emerges and suggesting individual shares based on a formula where a player gets back his marginal contribution, which is the portion of the collective income that was earned owed to his joining the group. The solidarity value introduces a change in which wealthy players (i.e., those holding large shares under the Shapley value) help less fortunate ones. In this paper, we want to understand how the solidarity value works and whether it properly addresses solidarity issues.
Firstly, we investigate on the fairness of the solidarity bills. An example shows a possible unwanted side effect of the solidarity value: a privileged player under the Shapley value $i$ pays solidarity bills to the extent of his good action setting him even poorer than another player $j$ who was poorer than $i$ and is now under the solidarity value better off thanks to receiving solidarity assistance. However, our main result here shows that this kind of injustice is rather limited in size, in a sense that we will clarify. That result holds for all TU games, and it states that the solidarity value always preserves the order in which any two players are ranked by the desirability relation.

Secondly, the following more specific questions also need answers:

(Q1) How accurately do solidarity gifts target the players of poorer condition?

(Q2) Of what amount are the bills of solidarity interventions and who settles them? Are the payers selected with respect to their wealth?

But our contribution to this issue fails to cover the scope of all general TU games. Instead, we study two classes of TU 
games that we obtain by modeling some real world business situations. The two classes of TU games are

(1) additive games in which a coalition's production is the sum of its members' individual productions,

(2) a model of an enterprise seen as a game where the enterprise offers to recruit workers, to turn their productivity into production (because it detains the logistic, the tools for production, the management framework, and other means), to pay them salaries withdrawn from the production (after subtracting production cost) and to keep the remaining funds for itself as its benefit. The worker's share in the side distribution is the salary that he gets, while the enterprise's share is what it keeps.

For the two classes of games, we are able to get full answers to the questions (Q1) and (Q2). Our approach is as follows: when the position of player $i$ in a given game of the above mentioned classes is fully determined by a quota $q_{i} \in \mathbb{R}$, we simulate a new game which is identical to that game except $q_{i}$ which is replaced with $q_{i}+\alpha, \alpha \in \mathbb{R}$. How then will the additional profit due to $\alpha$ be shared among the players? After processing the transformed game through Nowak and Radzik's formula, we watch out for the variations of shares due to $\alpha$ in the Shapley value compared to the variations in the solidarity value. This unveils transfers of additional funds done by player $i$ under the solidarity value and transfers under the Shapley value, and we thereby capture the solidarity reflex induced on player $i$ by the solidarity value context.

This introduction is followed by Section 2 which recalls the Shapley and the solidarity values. Section 3 discusses a general concern of fairness when performing solidarity assistance. A theorem on the limited size of possible injustice under the solidarity value is obtained for general TU games. Sections 4 and 5 study additive games and a model of enterprise, respectively, to unveil all flows of solidarity funds under the solidarity value and bring full answers to questions (Q1) and (Q2) about these particular classes of games. Here, we also obtain formulas for computing individual shares alongside with the various player to player solidarity transfers. Section 6 concludes the work.

\section{A Recall of the Shapley Value and the Solidarity Value}

A transferable utility game (or TU game) is any $(N, v)$ where $N=\{1,2, \ldots, n\}$ is the set of players and $v$ is an application which associates any $S \subseteq N$ with a real number $v(S)$ with $v(\varnothing)=0$. The members of $S$ will produce an income measured by $v(S)$, provided that they join efforts and act in coordination.

Let us first recall the Shapley and the solidarity values. The following quantities are used (where $i$ is a player and $T$ a nonempty coalition):

$$
\begin{gathered}
A_{i}^{v}(T)=v(T)-v(T \backslash\{i\}), \\
B^{v}(T)=\frac{1}{|T|} \sum_{k \in T}[v(T)-v(T \backslash\{k\})] .
\end{gathered}
$$

The number $A_{i}^{v}(T)$ is player $i$ 's marginal contribution to the coalition $T$, whereas $B^{v}(T)=(1 /|T|) \sum_{k \in T}[v(T)-v(T \backslash$ $\{k\})$ ] is the average marginal contribution of all members of $T$.

The Shapley value and the solidarity value assigned to every player $i$ are then defined, respectively, by $\mu_{i}(v)$ and $\Psi_{i}(v)$ given as follows:

$$
\begin{aligned}
& \mu_{i}(v)=\sum_{T \subseteq N / i \in T} \frac{(|T|-1) !(N \backslash T) !}{n !} A_{i}^{v}(T), \\
& \Psi_{i}(v)=\sum_{T \subseteq N / i \in T} \frac{(|T|-1) !(N \backslash T) !}{n !} B^{v}(T) .
\end{aligned}
$$

We will need another expression of $\mu_{i}(v)$ and $\Psi_{i}(v)$. So, let $\pi=\pi_{1} \pi_{2} \cdots \pi_{n}$ be a ranking of all players in a strict order where $\pi_{1}$ is the first, $\pi_{2}$ is the second,..., and $\pi_{n}$ is the last player, and let $S_{N}$ denote the set of all such rankings. The cardinality of $S_{N}$ is $\left|S_{N}\right|=n$ !. So, if a random draw with equal probability is conducted in $S_{N}$, then the chance of selecting each $\pi \in S_{N}$ is $1 / n !$. If $i$ is a player and $\pi \in S_{N}$, then there exists a unique $p \in\{1,2, \ldots, n\}$ called the rank of $i$ with respect to $\pi$ and denoted by $\operatorname{rank}_{\pi}(i)$ such that $i=\pi_{p}$.

For every $p \in\{1,2, \ldots,|N|\}$ and $\pi \in S_{N}$, also adopt the following notations:

$$
\begin{aligned}
& \pi_{\leq p}=\left\{\pi_{1}, \pi_{2}, \ldots, \pi_{p}\right\}, \quad \pi_{<p}=\pi_{\leq p} \backslash\left\{\pi_{p}\right\}, \quad \pi_{\leq 0}=\pi_{<1}=\varnothing . \\
& \widehat{\mu}_{i}(\pi)=\widehat{\mu}_{i}^{v}(\pi)=v\left(\pi_{\leq \operatorname{rank}_{\pi}(i)}\right)-v\left(\pi_{\leq \operatorname{rank}_{\pi}(i)} \backslash\{i\}\right) . \\
& \widehat{\Psi}_{i}(\pi)=\widehat{\Psi}_{i}^{v}(\pi)=\sum_{q=\operatorname{rank}_{\pi}(i)}^{n} \frac{1}{q}\left[v\left(\pi_{\leq q}\right)-v\left(\pi_{\leq q-1}\right)\right] .
\end{aligned}
$$

Here, $\widehat{\mu}_{i}(\pi)$ may be interpreted as the gain of $i$ if the players all join the grand coalition in a process where they come in one after another following a list described by $\pi$ (so, player $i$ enters at position $\operatorname{rank}_{\pi}(i)$ to join $\left.\pi_{\leq \operatorname{rank}_{\pi}(i)} \backslash\{i\}\right)$, where it is agreed that what each one gets is his marginal contribution to $\pi_{\leq \operatorname{rank}_{\pi}(i)}$, which is $v\left(\pi_{\leq \operatorname{rank}_{\pi}(i)}\right)-v\left(\pi_{\leq \operatorname{rank}_{\pi}(i)} \mid\right.$ $\{i\})$.

Player $i$ receives $\widehat{\Psi}_{i}(\pi)$ under the same mode of admission, but with the rule that $\widehat{\Psi}_{i}(\pi)$ is instead his share when the marginal contribution is distributed in equal shares between him and the players that have preceded him in the admission list.

The following statement presents well-known expressions of the Shapley and solidarity values where permutations play an important role.

Proposition 1. Let $(N, v)$ be TU game. Then for every $i \in N$, one has

$$
\begin{aligned}
& \text { (1) } \mu_{i}(v)=\sum_{\pi \in S_{N}} \frac{1}{n !} \widehat{\mu}_{i}(\pi) \\
& \text { (2) } \Psi_{i}(v)=\sum_{\pi \in S_{N}} \frac{1}{n !} \widehat{\Psi}_{i}(\pi) .
\end{aligned}
$$


TABLE 1

\begin{tabular}{lccccccc}
\hline$S$ & 1 & 2 & 3 & 12 & 13 & 23 & 123 \\
\hline$v(S)$ & 0 & 0 & 0 & 1 & 0 & 0 & 1 \\
\hline
\end{tabular}

If a random draw with equal chance is conducted in the set $S_{N}$, then the probability that a given $\pi \in S_{N}$ is selected is $1 / n$ !. The expression $\mu_{i}(v)=\sum_{\pi \in S_{N}}(1 / n !) \widehat{\mu}_{i}(\pi)$ is used to interpret $\mu_{i}(v)$ as the expected payoff (as usually understood by probabilists) of player $i$ when he keeps his marginal contribution for himself alone. The formula $\Psi_{i}(v)=$ $\sum_{\pi \in S_{N}}(1 / n !) \widehat{\Psi}_{i}(\pi)$ also presents $\Psi_{i}(v)$ as an expected payoff of player $i$ when the marginal contribution is instead shared in equal parts among the newcomer and those welcoming him.

These expressions of $\mu$ and $\Psi$ will play a crucial role in the sequel when we will analyze transfers of funds, because they break down the total gain of a player into elementary parts, each of which is related to a corresponding permutation $\pi$.

\section{About the Fairness of Solidarity Actions in a General TU Game}

Let us now investigate on how a player in poor condition may receive help from the others under the solidarity value. The following example was provided by Radzik and Nowak (1994).

Example 2. Let $N=\{1,2,3\}$, and $v$ is given in Table 1 .

In this game, player 3 is considered a disabled, because his joining or leaving any coalition has no effect on the productivity of that coalition. Radzik and Nowak compute the individual gains from the Shapley value, which are regarded as normal gains owed to individual merit. They obtain $\mu(v)=(1 / 2,1 / 2,0)$. So, player 3 is attributed 0 by the Shapley value, because each player keeps for himself his merited gain. As for the solidarity value, the gains are $\Psi(v)=(7 / 18,7 / 18,4 / 18)$, and we need to keep a record of the changes and appreciate their relevance. Under the idea of solidarity, player 3 receives some wealth amounting to $4 / 18$. The bill of that gift has been paid in equal contribution by players 1 and 2 who pay 2/18 each. Remark that, in this example, the players' ranking with respect to their gains under the Shapley value is not changed under the solidarity value. Moreover, the gap between each player and any richer one has been reduced in $\Psi(v)$, after all solidarity actions have been processed. In the next section, we naturally wonder whether the fairness illustrated in this example will always prevail under the solidarity value.

In a TU game, take a player $i$ with a better condition than a fellow player $j$ according to merit attested by the Shapley value, that is $\mu_{i}(v)>\mu_{j}(v)$. Of course, there is no clear-cut idea of what amount of solidarity help player $i$ should pay to $j$, neither is there any moral consideration commanding that $i$ assists $j$ only because $\mu_{i}(v)>\mu_{j}(v)$. However, a "large" inequality (i.e., when the difference $\mu_{i}(v)-\mu_{j}(v)$ is considered to be big) is a major issue of concern. Of course, player $j$ is not expected to enjoy such a situation. This anomaly of large player to player inequalities may occur for other pairs of players than $i$ and $j$, to the extent of causing social malaise. Inequality indices are meant to tell to what extent the entire community is affected. A call for solidarity would normally invite a rich player to transfer part of his wealth to a poor one. The quantity $\delta$ that player $i$ with $\mu_{i}(v)>\mu_{j}(v)$ needs to transfer to player $j$ should be large enough to "narrow" the inequality by reducing the difference $\mu_{i}(v)-\delta-\left(\mu_{j}(v)+\delta\right)$ to a bearable value and at the same time moderate enough to avoid the injustice of creating an inverse inequality that will instead favor $j$. Since there may exist many rich people and also many poor ones, the search for right player to player transfers that are likely to solve the issue is an important challenge. The idea of Pigou-Dalton transfers (Pigou [3] and Dalton [4]) recommends that transfers should be at the same time inequality narrowing and rank preserving. It is usually necessary to get through progressively, that is, by operating a final number of successive steps. For recent literature on Pigou-Dalton transfers, see Ebert [5] and Chameni Nembua and Themoi [6].

Radzik and Nowak show that the Shapley value fails to address solidarity concerns, and the solidarity value addresses that shortcoming. A different approach (e.g., by Pigou-Dalton transfers) will reduce inequality and keep the ranks of players but may ignore the production process modelled by the characteristic function $v$. Instead, the policy for sharing every marginal contribution is modified. This adjustment from within where merely one point in the rules of the game changes is remarkably interesting. Indeed, the change is unartificial and self-implementing since it is embedded into the players' interaction setting. Nevertheless, we need to appreciate the end result. To that purpose, we consider the solidarity value as a result of player to player transfers of funds operated on the Shapley value, and we will investigate the fairness of those transfers.

First of all, what is our idea of fairness about? If $\mu_{i}(v)>$ $\mu_{j}(v)$, we are focused on how the solidarity value may increase player $j$ 's share at the expense of $i$, on the grounds of solidarity fellowship, to adopt the new shares $\Psi_{i}(v)$ and $\Psi_{j}(v)$. Of course, we would therefore have $\mu_{i}(v)>\Psi_{i}(v)$ and $\Psi_{j}(v)>\mu_{j}(v)$. To avoid injustice, $\Psi$ should at least preserve the relative ranks of the two players with respect to $\mu$ (i.e., $\Psi_{i}(v)>$ $\Psi_{j}(v)$, since we already have $\left.\mu_{i}(v)>\mu_{j}(v)\right)$, and we even tolerate $\Psi_{i}(v)=\Psi_{j}(v)$. To summarize, we naturally expect to have $\mu_{i}(v)>\Psi_{i}(v) \geq \Psi_{j}(v)>\mu_{j}(v)$, which is absolutely necessary. But are we protected against the shortcoming of having $\Psi_{i}(v)<\Psi_{j}(v)$ ? May paying player $i$ 's solidarity bills to $j$ according to Nowak and Radzik's formula reverse $i$ 's condition and lead to $\Psi_{i}(v)<\Psi_{j}(v)$ ? The following example tells us that, unfortunately, the answer is yes.

Example 3. Let $N=\{1,2,3\}$. The table of game is Table 2 .

The allocations are displayed in Table 3 (Table 3(b) is only for decimal approximation).

Player 1 tops the Shapley value's ranking with $\mu_{1}(v)=$ 0.70 , and player 2 is last with $\mu_{2}(v)=0.65$, a place which he shares with player 3 . In the switch for the solidarity value, player 1 pays $0.70-0.66=0.04$ while player 3 pays 
TABLE 2

\begin{tabular}{lccccccc}
\hline$S$ & 1 & 2 & 3 & 12 & 13 & 23 & 123 \\
\hline$v(S)$ & $1 / 10$ & 1 & 0 & 1 & 2 & 1 & 2 \\
\hline
\end{tabular}

TABle 3: (a) Exact allocations. (b) Approximate allocations.

(a)

\begin{tabular}{lccc}
\hline & \multicolumn{3}{c}{ Player $i$} \\
& 1 & 2 & 3 \\
\hline$\mu_{i}(v)$ & $14 / 20$ & $13 / 20$ & $13 / 20$ \\
$\Psi_{i}(v)$ & $236 / 360$ & $257 / 360$ & $227 / 360$ \\
\hline
\end{tabular}

(b)

\begin{tabular}{lccc}
\hline & \multicolumn{3}{c}{ Player $i$} \\
& 1 & 2 & 3 \\
\hline$\mu_{i}(v)$ & 0.70 & 0.65 & 0.65 \\
$\Psi_{i}(v)$ & 0.66 & 0.71 & 0.63 \\
\hline
\end{tabular}

$0.65-0.63=0.02$. Player 2 gets $0.71-0.65=0.06$ and he is raised to the top position. The main loser in the operation is player 1 who pays the largest portion of the bill. He reverses his condition and he now comes behind player 2. In this example, solidarity help prescribed by the solidarity value is exaggerated and is not rank-preserving. Also, it is absurd that player 3 pays some help to player 2 , while we have $\mu_{2}(v)=$ $\mu_{3}(v)$.

However, we will prove that this shortcoming (of a player swapping his privileged position with that of a poorer one to whom he has paid assistance) of the solidarity value is, in a sense that we explain, of limited size. To that purpose, let us adopt a way of telling which player $i$ enjoys a better position than which other one $j$. It seems natural to consider $i$ as being at least as productive as $j$ if $i$ 's marginal contribution to any coalition with $S \cap\{i, j\}=\varnothing$ is not less than $j$ 's marginal contribution to the same coalition. This is written by $v(S \cup\{i\})-v(S) \geq v(S \cup\{j\})-v(S)$, or in a much simpler way $v(S \cup\{i\}) \geq v(S \cup\{j\})$. So, the relation $\geq$ on $N$ is defined as follows.

Definition 4. Let $(N, v)$ be a TU game and $i, j \in N$ with $i \neq j$. The desirability relation is the relation $\geq$ defined on $N$ by

$$
(i \geq j) \Longleftrightarrow[\forall S \subseteq N \backslash\{i, j\}, v(S \cup\{i\}) \geq v(S \cup\{j\})]
$$

The above relation is a preorder in simple games, called the desirability relation, and its strict part is denoted by $>$. It is widely used in the literature, mostly to evaluate individual influence in a voting committee modelled by a simple game. See Isbell (1958) [7], Taylor and Zwicker ((1993) [8], (1999) [9]), Diffo Lambo and Moulen (2002) [10], Friedman et al. (2006) [11], and Carreras and Freixas (2008) [12]. Also see Tchantcho et al. (2008) [13] for extensions to more general committees. This relation has also been transposed to TU games, written exactly with the formula above and referred to as the standard desirability relation by Freixas (2010) [14]. Also refer to Freixas (2010) for the latest complete roundup of the literature on how players in a TU game may be compared using a binary relation.

It is easy to prove that the desirability relation is a subpreorder of the preorder induced on $N$ by $\mu$. This means that the following is true: for every $i, j \in N$ with $i \neq j$,

$$
(i>j) \Longleftrightarrow[(i \geq j) \text { and non }(j \geq i)]
$$

The following result states that the desirability relation is a subpreorder of the preorder induced on $N$ by $\Psi$. Therefore the primacy of $i$ over $j$ acknowledged by the desirability relation is not reversed under the solidarity value. So, the type of "injustice" illustrated in Example 3 is bounded in the sense that a player with a better position than another player in a sense at least as strong as the desirability relation still holds a better position after the settlement of all solidarity bills.

Theorem 5. Let $(N, v)$ be a TU game and $i, j \in N$ with $i \neq j$. Then one has

$$
\begin{aligned}
& \text { (1) }(i \succeq j) \Rightarrow\left[\Psi_{i}(v) \geq \Psi_{j}(v)\right], \\
& \text { (2) }(i \succ j) \Rightarrow\left[\Psi_{i}(v)>\Psi_{j}(v)\right] .
\end{aligned}
$$

Proof. For every $k \in N$, and $\pi \in S_{N}$, denote by $\operatorname{rank}_{\pi}(k)$ the unique $p \in\{1,2, \ldots, n\}$ such that $\pi_{p}=k$.

Let $A=\left\{\pi \in S_{N} / \operatorname{rank}_{\pi}(i)<\operatorname{rank}_{\pi}(j)\right\}$ and $B=\{\pi \in$ $\left.S_{N} / \operatorname{rank}_{\pi}(i)>\operatorname{rank}_{\pi}(j)\right\}$. For every $\pi \in S_{N}$, define $\pi^{i j}=$ $\pi_{1}^{i j} \pi_{2}^{i j} \cdots \pi_{n}^{i j} \in S_{N}$ by the following: for every $s \in\{1,2, \ldots, n\}$,

$$
\pi_{s}^{i j}= \begin{cases}\pi_{s} & \text { if } \pi_{s} \notin\{i, j\} \\ i & \text { if } \pi_{s}=j \\ j & \text { if } \pi_{s}=i\end{cases}
$$

Then, $\forall \pi \in S_{N}, \pi \in A \Leftrightarrow \pi^{i j} \in B, S_{N}=A \cup B$, and $A \cap B=$ $\varnothing$. Therefore, $\Psi_{i}(v)=\sum_{\pi \in S_{N}} \widehat{\Psi}_{i}(\pi)=\sum_{\pi \in A}\left[\widehat{\Psi}_{i}(\pi)+\widehat{\Psi}_{i}\left(\pi^{i j}\right)\right]$. Let us assume that $i \geq j$, and let us first prove that $\forall \pi \in A$, $\widehat{\Psi}_{i}(\pi)+\widehat{\Psi}_{i}\left(\pi^{i j}\right) \geq \widehat{\Psi}_{j}(\pi)+\widehat{\Psi}_{j}\left(\pi^{i j}\right)$. For every $\pi \in A$, let $p, q \epsilon$ $\{1,2, \ldots, n\}$ such that $p=\operatorname{rank}_{\pi}(i)$ and $q=\operatorname{rank}_{\pi}(j)$. Then we have $p<q$ and $q=\operatorname{rank}_{\pi^{i j}}(i)$. Therefore, with $R_{i}=\widehat{\Psi}_{i}(\pi)+$ $\widehat{\Psi}_{i}\left(\pi^{i j}\right)$ and $R_{j}=\widehat{\Psi}_{j}(\pi)+\widehat{\Psi}_{j}\left(\pi^{i j}\right)$,

$$
\begin{aligned}
R_{i}= & \sum_{s=p}^{n} \frac{1}{s}\left[v\left(\pi_{\leq s}\right)-v\left(\pi_{<s}\right)\right] \\
& +\sum_{s=q}^{n} \frac{1}{s}\left[v\left(\pi_{\leq s}^{i j}\right)-v\left(\pi_{<s}^{i j}\right)\right] \\
= & \sum_{s \leq p<q} \frac{1}{s}\left[v\left(\pi_{\leq s}\right)-v\left(\pi_{<s}\right)\right] \\
& +\sum_{q \leq s \leq n} \frac{1}{s}\left[v\left(\pi_{\leq s}\right)-v\left(\pi_{<s}\right)\right] \\
& +\sum_{q \leq s \leq n} \frac{1}{s}\left[v\left(\pi_{\leq s}^{i j}\right)-v\left(\pi_{<s}^{i j}\right)\right],
\end{aligned}
$$




$$
\begin{aligned}
R_{j}= & \sum_{s=q}^{n} \frac{1}{s}\left[v\left(\pi_{<s} \cup\left\{\pi_{s}\right\}\right)-v\left(\pi_{<s}\right)\right] \\
& +\sum_{s=p}^{n} \frac{1}{s}\left[v\left(\pi_{<s}^{i j} \cup\left\{\pi_{s}^{i j}\right\}\right)-v\left(\pi_{<s}^{i j}\right)\right] \\
= & \sum_{q \leq s \leq n} \frac{1}{s}\left[v\left(\pi_{<s} \cup\left\{\pi_{s}\right\}\right)-v\left(\pi_{<s}\right)\right] \\
& +\sum_{p \leq s<q} \frac{1}{s}\left[v\left(\pi_{\leq s}^{i j}\right)-v\left(\pi_{<s}^{i j}\right)\right] \\
& +\sum_{q \leq s \leq n} \frac{1}{s}\left[v\left(\pi_{\leq s}^{i j}\right)-v\left(\pi_{<s}^{i j}\right)\right] .
\end{aligned}
$$

Therefore, we only need to prove that $a_{i} \leq a_{j}$, where

$$
\begin{aligned}
& a_{i}=\sum_{s \leq p<q} \frac{1}{s}\left[v\left(\pi_{\leq s}\right)-v\left(\pi_{<s}\right)\right], \\
& a_{j}=\sum_{p \leq s<q} \frac{1}{s}\left[v\left(\pi_{\leq s}^{i j}\right)-v\left(\pi_{<s}^{i j}\right)\right] .
\end{aligned}
$$

Remark that

$$
\begin{aligned}
a_{i}= & \frac{1}{q-1} v\left(\pi_{\leq q-1}\right)-\frac{1}{p} v\left(\pi_{<p}\right) \\
& +\sum_{p \leq s<q-1} \frac{1}{(s+1) s} v\left(\pi_{\leq s}\right), \\
a_{j}= & \frac{1}{q-1} v\left(\pi_{\leq q-1}^{i j}\right)-\frac{1}{p} v\left(\pi_{<p}^{i j}\right) \\
& +\sum_{p \leq s<q-1} \frac{1}{(s+1) s} v\left(\pi_{\leq s}^{i j}\right) .
\end{aligned}
$$

Indeed, we have

$$
\begin{aligned}
a_{i} & =\sum_{p \leq s<q} \frac{1}{s}\left[v\left(\pi_{\leq s}\right)-v\left(\pi_{<s}\right)\right] \\
& =\sum_{p \leq s<q} \frac{1}{s} v\left(\pi_{\leq s}\right)-\sum_{p \leq s<q} \frac{1}{s} v\left(\pi_{<s}\right) \\
& =\sum_{p \leq s<q} \frac{1}{s} v\left(\pi_{\leq s}\right)-\sum_{p \leq s+1<q} \frac{1}{s+1} v\left(\pi_{<s+1}\right) \\
& =\sum_{p \leq s<q} \frac{1}{s} v\left(\pi_{\leq s}\right)-\sum_{p-1 \leq s<q-1} \frac{1}{s+1} v\left(\pi_{\leq s}\right) \\
& =\sum_{p \leq s<q-1} \frac{1}{s} v\left(\pi_{\leq s}\right)+\frac{1}{q-1} v\left(\pi_{\leq q-1}\right) \\
& -\frac{1}{p} v\left(\pi_{\leq p-1}\right)-\sum_{p \leq s<q-1} \frac{1}{s+1} v\left(\pi_{\leq s}\right)
\end{aligned}
$$

$$
\begin{gathered}
=\frac{1}{q-1} v\left(\pi_{\leq q-1}\right)-\frac{1}{p} v\left(\pi_{\leq p-1}\right) \\
+\sum_{p \leq s<q-1}\left(\frac{1}{s}-\frac{1}{s+1}\right) v\left(\pi_{\leq s}\right) .
\end{gathered}
$$

This proves the formula for $a_{i}$, and the expression for $a_{j}$ is a straightforward consequence. We deduce that

$$
\begin{aligned}
a_{i}-a_{j}= & \frac{1}{q-1}\left[v\left(\pi_{\leq q-1}\right)-v\left(\pi_{\leq q-1}^{i j}\right)\right] \\
& -\frac{1}{p}\left(v\left(\pi_{<p}\right)-v\left(\pi_{<p}^{i j}\right)\right) \\
& +\sum_{p \leq s<q-1} \frac{1}{(s+1) s}\left[v\left(\pi_{\leq s}\right)-v\left(\pi_{\leq s}^{i j}\right)\right] .
\end{aligned}
$$

But $\pi_{<p}=\pi_{<p}^{i j}$, and we have $v\left(\pi_{<p}\right)-v\left(\pi_{<p}^{i j}\right)=0$. So,

$$
\begin{aligned}
a_{i}-a_{j}= & \frac{1}{q-1}\left[v\left(\pi_{\leq q-1}\right)-v\left(\pi_{\leq q-1}^{i j}\right)\right] \\
& +\sum_{p \leq s<q-1} \frac{1}{(s+1) s}\left[v\left(\pi_{\leq s}\right)-v\left(\pi_{\leq s}^{i j}\right)\right] .
\end{aligned}
$$

But when $p \leq s<q-1$, we have $\pi_{\leq s}^{i j}=\left(\pi_{\leq s} \backslash\{i, j\}\right) \cup\{j\}$ and $\pi_{\leq s}=\left(\pi_{\leq s} \backslash\{i, j\}\right) \cup\{i\}$. Therefore, $v\left(\pi_{\leq s}\right) \geq v\left(\pi_{\leq s}^{i j}\right)$ since $i \geq j$. We deduce that $a_{i}-b_{j} \geq 0$. Therefore, $\Psi_{i}(v) \geq \Psi_{j}(v)$.

Now, assume that $i \succ j$. Then we have non $\forall S \subseteq N \backslash\{i, j\}$, $v(S \cup\{j\}) \geq v(S \cup\{i\})$. Therefore, there exists $S \subseteq N \backslash\{i, j\}$ such that $v(S \cup\{i\})>v(S \cup\{j\})$. Let $|S|=p-1$, and $S=$ $\left\{\pi_{1}, \pi_{2}, \ldots, \pi_{p-1}\right\}, \pi_{p}=i, \pi_{p+1}=j$ and $N \backslash[S \cup\{i, j\}]=$ $\left\{\pi_{p+1}, \pi_{p+2}, \ldots, \pi_{n}\right\}$. Define $\pi \in S_{N}$ by $\pi=\pi_{1} \pi_{2} \cdots \pi_{n}$. In this $\pi, i$ holds the rank $q=p+1$. It is sufficient to show that, for this $\pi, a_{i}$ and $a_{j}$ in the proof of Theorem 5 satisfy the strict inequality $a_{i}-a_{j}>0$. In fact, the term for $s=p$ in the expression of $a_{i}-a_{j}$ above is $(1 /(p+1) p)\left[v\left(\pi_{\leq p}\right)-v\left(\pi_{\leq p}^{i j}\right)\right]$. Also, we have $\pi_{\leq p}=\pi_{<p} \cup\left\{\pi_{p}\right\}=S \cup\{i\}$ and $\pi_{\leq p}^{i j}=\pi_{<p}^{i j} \cup\left\{\pi_{p}^{i j}\right\}=$ $S \cup\{j\}$, and we are through, since $v(S \cup\{i\})>v(S \cup\{j\})$.

In a general TU game $(N, v)$, we may have $\mu_{i}(v)>\mu_{j}(v)$ or $i>j$. These inequalities are two ways of modeling the "degree" with which player $i$ "dominates" $j$. But we know that $(i>j) \Rightarrow\left(\mu_{i}(v)>\mu_{j}(v)\right)$. So, $i>j$ states that $i$ dominates $j$ in a sense that is stronger than just having $\mu_{i}(v)>\mu_{j}(v)$ which is weaker. Example 3 has shown that solidarity reflected by $\Psi$ is sometimes unfair, since we may have $\mu_{i}(v)>\mu_{j}(v)$ and end up with this dominance being opposed by the solidarity value with $\Psi_{i}(v)<\Psi_{j}(v)$. However, the above theorem tells that this unfairness is not too exaggerated, since it states that if $\mu_{i}(v)>\mu_{j}(v)$ to a high extent where $i$ 's dominance is condoned by the desirability relation (i.e., $i>j$ ), then the solidarity value complies and we have $\Psi_{i}(v)>\Psi_{j}(v)$. So one has the following.

(1) If $\mu_{i}(v)>\mu_{j}(v)$, then $i$ dominates $j$ weakly, and the solidarity value sometimes opposes it and we may have $\Psi_{i}(v)<\Psi_{j}(v)$. 
(2) If $i>j$, then $i$ dominates $j$ in a sense stronger than in (1) above and the solidarity value complies so that we have $\Psi_{i}(v)>\Psi_{j}(v)$.

In order to state a straightforward corollary of Theorem 5, we need to recall the definition of weak desirability relation $\succeq_{d}$ (with the strict part denoted by $\succ_{d}$ ) which is a preorder on $N$ that generalizes the desirability relation. So, $\succeq_{d}$ is defined as follows: $\left(i \succeq_{d} j\right) \Leftrightarrow \forall k \in\{0,1, \ldots, n-1\}$,

$$
\begin{aligned}
& \sum_{S \subseteq N \backslash\{i\},|S|=k}[v(S \cup\{i\})-v(S)] \\
& \geq \sum_{S \subseteq N \backslash\{j\},|S|=k}[v(S \cup\{j\})-v(S)] .
\end{aligned}
$$

The desirability relation is a subpreorder of the weak desirability relation (i.e., $\forall i, j \in N, i \geq j \Rightarrow i \succeq_{d} j$ and $i>j \Rightarrow i \succ_{d} j$ ). There exists a class of values that comprises the Shapley value called regular semivalues. It is shown (Freixas, 2010) that given any TU game $(N, v), \succeq_{d}$ is complete if and only if the preorders induced on $N$ by all regular semivalues coincide with the desirability relation. The reader may report to Freixas (2010) for the notions of semivalues, regular semivalues, and more on TU games and conditions under which the preorders induced by different values may coincide. Carreras and Freixas (2008) provide similar investigations for simple games. Hence, when the TU game is linear (i.e., $\succeq$ is complete), then $\succeq$ coincides with the weak desirability relation, and with all preorders induced by regular semivalues as well. We then deduce from Theorem 5 that if this is the case, the preorder induced by $\Psi$ and the preorders induced by all regular semivalues coincide with the desirability relation.

Corollary 6. If the TU game is linear, then the preorders induced on the set of players by the solidarity value and all regular semivalues coincide.

\section{Solidarity Transfers in Additive Games}

People in $N=\{1,2, \ldots, n\}$ who create a firm assemble their shares which we model by $q=\left(q_{i}\right)_{i \in N}$ for the capital of that firm. We model the profit making environment by a TU game $(N, v)$ where it is assumed that a business run by $S \subseteq N$ will produce profit which is quantified by $v(S) \in \mathbb{R}$. We assume that profit is proportional to capital throughout the market. As a real world illustration for this assumption, consider the environment created by a state firm called SODECOTON in the northern province of Cameroon which buys cotton at a fixed price per kilogram from the local peasants for exportation. The government always ensures that SODECOTON buys the total local production irrespective of whatever happens during exportation. Any group of peasants may create their firm by contributing shares for renting the land on which they will collectively grow cotton and for funding the labor costs and all other production expenses. Naturally, the size of the land, the labor costs, the expenses for growing the cotton, the quantity of their production, and the total sales revenue are each proportional to the amount of money at the input. Therefore in this context of cultivating cotton for SODECOTON, profit is proportional to capital.

Back to the general case, let $\tau$ be the profit rate. A constituted coalition $S \subseteq N$ invests the capital $\sum_{i \in S} q_{i}$ to gain the surplus $v(S)=P\left(\sum_{i \in S} q_{i}\right)$ which is such that $\tau=P\left(\sum_{i \in S} q_{i}\right) / \sum_{i \in S} q_{i}$ does not vary with $S$, since we are assuming that profit is proportional to capital. We deduce that $P\left(\sum_{i \in S} q_{i}\right)=\left(\sum_{i \in S} q_{i}\right) \tau$ and we can therefore adopt the following formula:

$$
\forall S \subseteq N, \quad v(S)=\left(\sum_{i \in S} q_{i}\right) \tau .
$$

This game is said to be additive because it satisfies $\forall S, T \subseteq$ $N$ with $S \cap T=\varnothing, v(S \cup T)=v(S)+v(T)$. So, an additive game is given by its sequence of "quotas" $q=\left(q_{1}, q_{2}, \ldots, q_{n}\right)$.

It is easy to compute the individual shares under the Shapley value, which are $\forall i \in N, \mu_{i}(v)=q_{i} \tau$. So, if they are on the side distribution according to the Shapley value, player $i$ keeps the profit brought about by his share $q_{i}$ which is $q_{i} \tau$. His property in the firm, which amounted to $q_{i}$ before the game then becomes $q_{i}+q_{i} \tau$ after. But under the solidarity value, some players will pay solidarity help, some others will get help, and computing individual shares will be less obvious.

4.1. Individual Shares under the Solidarity Value in Additive Games. We will not address the given game $v$ with quotas $q=\left(q_{i}\right)_{i \in N}$ directly, because it will be less tedious to get to $v$ by successive transformations of games starting from the null game $v_{0}$ with quotas $(0,0, \ldots, 0)$. Then we will successively move through the following games: $v_{1}=T_{1}^{q_{1}}\left(v_{0}\right)$ with quotas $\left(q_{1}, 0, \ldots, 0\right), v_{2}=T_{2}^{q_{2}}\left(v_{1}\right)$ with quotas $\left(q_{1}, q_{2}, \ldots, 0\right), \ldots, v_{n}=$ $T_{n}^{q_{n}}\left(v_{n-1}\right)$ with quotas $\left(q_{1}, q_{2}, \ldots, q_{n}\right)$. To that purpose, let us first define the $T^{\alpha}$-transformation of any additive game as follows.

Definition 7 . Let $(N, v)$ be an additive game with quotas $q=$ $\left(q_{1}, q_{2}, \ldots, q_{n}\right)$; take $i \in N$, and $\alpha \in \mathbb{R}$. The $T_{i}^{\alpha}$-transformation of $(N, v)$ is the new additive game $\left(N, v^{\alpha}\right)$ with quotas $q^{\alpha}=$ $\left(q_{1}^{\alpha}, q_{2}^{\alpha}, \ldots, q_{n}^{\alpha}\right)$ where

$$
\forall k \in N, \quad q_{k}^{\alpha}= \begin{cases}q_{k} & \text { if } k \neq i \\ q_{k}+\alpha & \text { if } k=i\end{cases}
$$

The $T_{i}^{\alpha}$-transformation models a simulation where the prevailing game is $(N, v)$ and all of a sudden, player $i$ picks a bonus (which may be regarded as a drop of additional fund $\alpha$ that increases his input share before investment) unlike the other players $j$ who simply keep their prevailing $q_{j}$. In this elementary case, we will learn about how solidarity works in the solidarity value by watching how additional profit due to the bonus $\alpha$ will be shared among the players. To that purpose, will express $\Psi_{j}\left(v^{\alpha}\right), j \in N$ in a formula that highlights the variation of $\Psi_{j}(v)$ owed to $\alpha$.

First, let $j=i$. We will see what player $i$ keeps for himself: for every $s \in\{1,2, \ldots, n\}$, let $E^{s}=\left\{\pi \in S_{N} / \pi_{s}=i\right\}$. Then we have the following which are straightforward:

(1) $s \neq s^{\prime} \Rightarrow E^{s} \cap E^{s^{\prime}}=\varnothing$, 
(2) $S_{N}=\bigcup_{s=1}^{n} E^{s}$,

(3) $\left|E^{s}\right|=(n-1)$ !.

Moreover, if $\pi \in E^{s}$, then $\pi_{s}=i$. So, we have $q_{\pi_{s}}^{\alpha}=q_{i}+\alpha$, while $(t>s+1) \Rightarrow q_{\pi_{t}}^{\alpha}=q_{\pi_{t}}$. So,

$$
\begin{aligned}
\widehat{\Psi}_{i}^{v^{\alpha}}(\pi) & =\sum_{t=s}^{n}\left(\frac{1}{t} q_{\pi_{t}}^{\alpha}\right) \tau \\
& =\left(\frac{1}{s} q_{\pi_{s}}^{\alpha}\right) \tau+\sum_{t=s+1}^{n}\left(\frac{1}{t} q_{\pi_{t}}^{\alpha}\right) \tau \\
& =\frac{1}{s}\left(q_{i}+\alpha\right) \tau+\tau \sum_{t=s+1}^{n} \frac{1}{t} q_{\pi_{t}} \\
& =\tau \frac{\alpha}{s}+\tau \frac{1}{s} q_{\pi_{s}}+\tau \sum_{t=s+1}^{n} \frac{1}{t} q_{\pi_{t}} \\
& =\frac{\alpha}{s} \tau+\widehat{\Psi}_{i}^{v}(\pi) .
\end{aligned}
$$

We now compute $\Psi_{i}\left(v^{\alpha}\right)$ as follows:

$$
\begin{aligned}
\Psi_{i}\left(v^{\alpha}\right) & =\sum_{\pi \in S_{N}} \widehat{\Psi}_{i}^{v^{\alpha}}(\pi) \\
& =\sum_{s=1}^{n}\left(\sum_{\pi \in E^{s}}\left(\widehat{\Psi}_{i}^{v}(\pi)+\frac{\alpha}{s} \tau\right)\right) \\
& =\sum_{s=1}^{n}\left(\sum_{\pi \in E^{s}} \widehat{\Psi}_{i}^{v}(\pi)+\left|E^{s}\right| \frac{\alpha \tau}{s}\right) \\
& =\left(\sum_{s=1}^{n} \sum_{\pi \in E^{s}} \widehat{\Psi}_{i}^{v}(\pi)+\sum_{s=1}^{n}\left|E^{s}\right| \frac{\alpha}{s} \tau\right) \\
& =\widehat{\Psi}_{i}^{v}+\alpha \tau \sum_{s=1}^{n}\left[(n-1) ! \frac{1}{s}\right] \\
& =\widehat{\Psi}_{i}^{v}+\tau \alpha(n-1) ! \sum_{s=1}^{n} \frac{1}{s} .
\end{aligned}
$$

Since $\Psi_{i}(v)=(1 / n !) \widehat{\Psi}_{i}^{v}$, and $(n-1) ! / n !=1 / n$ therefore with $\gamma(n)=(1 / n)\left[\sum_{s=1}^{n}(1 / s)\right]$, we have

$$
\Psi_{i}\left(v^{\alpha}\right)=\Psi_{i}(v)+\gamma(n) \alpha \tau .
$$

This means that, out of the bonus $\alpha$, player $i$ keeps $\gamma(n) \alpha \tau$ for himself while the others get $(1-\gamma(n)) \alpha \tau$ to be shared.

Remark that if $n=1$, then $N=\{i\}$ and $\gamma(n)=1$. So, the formula (R1) becomes $\Psi_{1}\left(v^{\alpha}\right)=\Psi_{1}(v)+\alpha \tau$, which simply means that the only player in the game keeps the additional gain $\alpha \tau$ due to $\alpha$ for himself. However, TU games with $n=1$ are degenerate and obvious cases with no challenge and no attention needed. From now on in this work, all TU games considered have at least two players.

Now, let $j \in N \backslash\{i\}$. We need to compute $\Psi_{j}\left(v^{\alpha}\right)$. For $\pi \in S_{N}$, having $\widehat{\Psi}_{j}^{v^{\alpha}}(\pi) \neq \widehat{\Psi}_{j}^{v}(\pi)$ means that, in $\pi$, player $j$ has nonnull share from $\alpha$. Under the solidarity value, this means that player $j$ precedes player $i$ in $\pi$. So,

$$
\left[\widehat{\Psi}_{j}^{v^{\alpha}}(\pi) \neq \widehat{\Psi}_{j}^{v}(\pi)\right] \Longleftrightarrow\left[\operatorname{rank}_{\pi}(j)<\operatorname{rank}_{\pi}(i)\right]
$$

Assume that $\left[\operatorname{rank}_{\pi}(j)<\operatorname{rank}_{\pi}(i)\right]$, let $r=\operatorname{rank}_{\pi}(j)$ and $t=\operatorname{rank}_{\pi}(i)$. Then we have

$$
\begin{aligned}
\widehat{\Psi}_{j}^{v^{\alpha}}(\pi) & =\sum_{s=r}^{n} \frac{1}{s} q_{\pi_{s}}^{\alpha} \tau \\
& =\frac{1}{t} q_{i}^{\alpha} \tau+\sum_{r \leq s \leq n ; s \neq t} \frac{1}{s} q_{\pi_{s}}^{\alpha} \tau \\
& =\frac{1}{t}\left[q_{i}+\alpha\right] \tau+\sum_{r \leq s \leq n ; s \neq t} \frac{1}{s} q_{\pi_{s}} \tau \\
& =\frac{\alpha}{t} \tau+\frac{q_{i}}{t} \tau+\sum_{r \leq s \leq n ; s \neq t} \frac{1}{s} q_{\pi_{s}} \tau \\
& =\frac{\alpha}{t} \tau+\widehat{\Psi}_{j}^{v}(\pi) \\
& =\frac{\alpha}{\operatorname{rank}_{\pi}(i)} \tau+\widehat{\Psi}_{j}^{v}(\pi) .
\end{aligned}
$$

So, we have

$$
\widehat{\Psi}_{j}^{v^{\alpha}}(\pi)=\frac{\alpha}{\operatorname{rank}_{\pi}(i)} \tau+\widehat{\Psi}_{j}^{v}(\pi)
$$

This means that, in the switch to $v^{\alpha}$, player $j \neq i$ gets either additional share 0 when $\operatorname{rank}_{\pi}(j)>\operatorname{rank}_{\pi}(i)$, or $\widehat{\Psi}_{j}^{v^{\alpha}}(\pi)-$ $\widehat{\Psi}_{j}^{v}(\pi)=\left(\alpha / \operatorname{rank}_{\pi}(i)\right) \tau$ when $\operatorname{rank}_{\pi}(j)<\operatorname{rank}_{\pi}(i)$. We also know that the number of $\pi \in S_{N}$ for which $\operatorname{rank}_{\pi}(j)<$ $\operatorname{rank}_{\pi}(i)$ does not vary with $j \neq i$. So, in the outcome, what $j$ gets does not vary with $j$.

But we have seen above that player $i$ leaves $(1-\gamma(n)) \alpha \tau$ to the other player in $N \backslash\{i\}$. So, the shares are equal in the distribution and each player $j \neq i$ will get $\Delta_{i}^{j}(\alpha)=((1-$ $\gamma(n)) /(n-1)) \alpha \tau$. So, we have $\forall j \neq i$,

$$
\Psi_{j}\left(v^{\alpha}\right)=\Psi_{j}(v)+\frac{1-\gamma(n)}{n-1} \alpha \tau
$$

We have the following result.

Proposition 8. When $n \geq 2$, the solidarity value for additive games with $q=\left(q_{i}\right)_{i \in N}$ is given by $\forall i \in N$,

$$
\Psi_{i}(v)=\gamma(n) q_{i} \tau+\frac{[1-\gamma(n)]}{n-1} \sum_{j \in N \backslash\{i\}} q_{j} \tau,
$$

where $\gamma(n)=(1 / n)\left[\sum_{s=1}^{n}(1 / s)\right]$.

Proof. Remark that $v=T_{n}^{q_{n}}\left(\cdots\left(T_{2}^{q_{2}}\left(T_{1}^{q_{1}}\left(v_{0}\right)\right)\right)\right)$ where $v_{0}$ is the null game. Now we apply $n$ iterations of the formulas (R1) and (R3) to conclude. 
4.2. Solidarity Flows in Additive Games. From the Shapley value, player $i$ gets $\mu_{i}(v)=q_{i} \tau$ as mentioned above. Under the solidarity value, he gets $\Psi_{i}(v)$. So, the difference from $\mu_{i}(v)$ to $\Psi_{i}(v)$, which is due to solidarity, is

$$
\Psi_{i}(v)-\mu_{i}(v)=-[1-\gamma(n)] q_{i} \tau+\frac{[1-\gamma(n)]}{n-1} \sum_{j \in N \backslash\{i\}} q_{j} \tau .
$$

From all that precedes, the solidarity flows of funds are as follows.

$(1-\gamma(n))$ is the fraction of $q_{i} \tau$ that player $i$ gives to the other players in $N \backslash\{i\}$ for an equal shares' distribution. Each gets the fraction $(1-\gamma(n)) /(n-1)$ of $q_{i} \tau$.

Each $j \in N \backslash\{i\}$ in turn transfers $([1-\gamma(n)] /(n-1)) q_{j} \tau$ to player $i$ as a solidarity gift.

In this context where every player gives solidarity contributions while receiving gifts from others, we wonder which players $i \in N$ really get help in the outcome, after all compensations. Equality (21) above can also be written as follows:

$$
\Psi_{i}(v)-\mu_{i}(v)=\left(-q_{i}+\frac{1}{n} \sum_{j \in N} q_{j}\right) \tau \frac{1-\gamma(n)}{n-1} n .
$$

There appears the threshold of access to solidarity consideration which is the average $q_{j}, j \in N$; that is $a=$ $(1 / n) \sum_{j \in N} q_{j}$.

(1) If $q_{i}=a$, then $\Psi_{i}(v)=\mu_{i}(v)$, and player $i$ enjoys no solidarity help in the outcome, neither does he pay out any.

(2) If $q_{i}<a$, then $\Psi_{i}(v)>\mu_{i}(v)$ so he receives some solidarity help which amounts to $((1-\gamma(n)) /(n-$ 1)) $n\left(a-q_{i}\right) \tau$

(3) If $q_{i}>a$, then $\Psi_{i}(v)<\mu_{i}(v)$, so he gives away some funds which amount to $\left(q_{i}-a\right)((1-\gamma(n)) /(n-1)) n \tau$ for solidarity contribution.

Therefore, only wealthy players pay solidarity contributions in the outcome. That contribution is proportional to $\left(q_{i}-a\right) \tau$, which may be regarded as a measure of the degree of wealthiness (null when $i$ is average, positive when $i$ is richer than average, and negative when $i$ is more indigent than average). The needy get solidarity help in the outcome which is proportional to $\left(a-q_{i}\right) \tau$ which may be regarded as a measure of the degree of indigence. This is a clear and simple illustration of how inequality is reduced through Erbert's principle of concentration (Ebert [5]).

\section{Solidarity Transfers in a Model of Enterprise}

The model of the above section on additive games is one aspect of an enterprise, seen as a game involving the shareholders. In this section, we want to model an enterprise as a game involving that enterprise and its employees. We have a community of individuals $\widetilde{N}=\{1,2, \ldots, n\}$. Each $i \in \widetilde{N}$ can produce a quantity of wealth measured by $q_{i} \in \mathbb{R}$, but the facilities for converting productivity to true production are detained by an institution $e$ which we call the enterprise, the firm, or the company. Player $i \in \widetilde{N}$ therefore needs to get recruited by $e$ as an "employee," so that, working for $e$, he will produce the quantity $q_{i}$. In our model, the enterprise is unique and has no competition with others to worry about. We model this environment by a TU game $(N, v)$ where $N=$ $\widetilde{N} \cup\{e\}, q_{e}=0$, and $v$ is defined by the following: for every $S \subseteq N$,

$$
v(S)= \begin{cases}0 & \text { if } e \notin S, \\ \sum_{i \in S} q_{i} & \text { if } e \in S .\end{cases}
$$

For us, $e$ is player $n+1$, and individual productivity profile is described in $N$ by $q=\left(q_{1}, q_{2}, \ldots, q_{n}, 0\right) \in \mathbb{R}^{N}$. The enterprise with no worker comes out yielding no production, while a player who is not recruited produces nothing.

5.1. Individual Shares under the Shapley Value in Our Model of Enterprise. Let us first select $i \in \widetilde{N}$. For motivations already discussed in the above section, we take $\alpha \in \mathbb{R}$, and we consider $q^{\alpha}=\left(q_{1}^{\alpha}, q_{2}^{\alpha}, \ldots, q_{n}^{\alpha}, 0\right)$ where, for every $s \in N, q_{s}+\alpha$ is defined as in Definition 7:

$$
q_{s}^{\alpha}= \begin{cases}q_{s}+\alpha & \text { if } s=i, \\ q_{s} & \text { if } s \neq i .\end{cases}
$$

(i) What portion of $\alpha$ does player $i$ keep for himself under the Shapley value?

Let $\pi \in S_{N}$, and let $r=\operatorname{rank}_{\pi}(i)$. Then we have $\widehat{\mu}_{i}^{v^{\alpha}}(\pi)=$ $v^{\alpha}\left(\pi_{\leq r}\right)-v^{\alpha}\left(\pi_{<r}\right)$.

If $r<\operatorname{rank}_{\pi}(e)$, then $e \notin \pi_{\leq r}$, and $v^{\alpha}\left(\pi_{\leq r}\right)=v^{\alpha}\left(\pi_{<r}\right)=$ $v\left(\pi_{\leq r}\right)=v\left(\pi_{<r}\right)=0$, so

$$
\begin{gathered}
\widehat{\mu}_{i}^{v^{\alpha}}(\pi)=v^{\alpha}\left(\pi_{\leq r}\right)-v^{\alpha}\left(\pi_{<r}\right)=0 ; \\
\hat{\mu}_{i}^{v}(\pi)=v\left(\pi_{\leq r}\right)-v\left(\pi_{<r}\right)=0 .
\end{gathered}
$$

If $r>\operatorname{rank}_{\pi}(e)$, then $e \in \pi_{\leq r}$, and we have

$$
\begin{aligned}
\widehat{\mu}_{i}^{v^{\alpha}}(\pi) & =v^{\alpha}\left(\pi_{\leq r}\right)-v^{\alpha}\left(\pi_{<r}\right) \\
& =\sum_{k \in \pi_{\leq r}} q_{k}^{\alpha}-\sum_{k \in \pi_{<r}} q_{k}^{\alpha} \\
& =q_{i}^{\alpha}=q_{i}+\alpha, \\
\widehat{\mu}_{i}^{v}(\pi) & =v\left(\pi_{\leq r}\right)-v\left(\pi_{<r}\right) \\
& =\sum_{k \in \pi_{\leq r}} q_{k}-\sum_{k \in \pi_{<r}} q_{k} \\
& =q_{i} .
\end{aligned}
$$

But the number of $\pi \in S_{N}$ with $\operatorname{rank}_{\pi}(e)<\operatorname{rank}_{\pi}(i)$ is $n ! / 2$; therefore the additional gain that $i$ keeps due to $\alpha$ is $\Delta_{i}^{\mu}=$ $\mu_{i}\left(v^{\alpha}\right)-\mu_{i}(v)=(n ! / 2)(\alpha / n !)=(1 / 2) \alpha$, so

$$
\mu_{i}\left(v^{\alpha}\right)=\mu_{i}(v)+\frac{1}{2} \alpha
$$


(ii) What portion of $\alpha$ does player $i$ transfer to the enterprise under the Shapley value? We now need to compute $\mu_{e}\left(v^{\alpha}\right)$.

Let $\pi \in S_{N}, r=\operatorname{rank}_{\pi}(e)$, and $t=\operatorname{rank}_{\pi}(i)$. Then

$$
\begin{array}{r}
\widehat{\mu}_{e}^{v^{\alpha}}(\pi)=v^{\alpha}\left(\pi_{\leq r}\right)-v^{\alpha}\left(\pi_{<r}\right)=\sum_{k \in \pi_{\leq r}} q_{k}^{\alpha}, \\
\widehat{\mu}_{e}^{v}(\pi)=v\left(\pi_{\leq r}\right)-v\left(\pi_{<r}\right)=\sum_{k \in \pi_{\leq r}} q_{k} \\
\text { since } e \in \pi_{\leq r}, \quad e \notin \pi_{<r} .
\end{array}
$$

If $\operatorname{rank}_{\pi}(e)<\operatorname{rank}_{\pi}(i)$, then $i \notin \pi_{\leq r}$. So, $\forall k \in \pi_{\leq r}, q_{k}^{\alpha}=q_{k}$, and we have $\widehat{\mu}_{e}^{\gamma^{\alpha}}(\pi)=\widehat{\mu}_{e}^{v}(\pi)$. So,

If $\operatorname{rank}_{\pi}(e)>\operatorname{rank}_{\pi}(i)$, then $i \in \pi_{\leq r}, e=\pi_{r}$, and $e \notin \pi_{<r}$.

$$
\begin{aligned}
\mu_{e}^{v^{\alpha}}(\pi) & =\sum_{k \in \pi_{\leq r}} q_{k}^{\alpha} \\
& =q_{i}^{\alpha}+\sum_{k \in \pi_{\leq r} ; k \neq i} q_{k}^{\alpha} \\
& =q_{i}+\alpha+\sum_{k \in \pi_{\leq r} ; k \neq i} q_{k}^{\alpha} \\
& =\mu_{e}^{v}(\pi)+\alpha .
\end{aligned}
$$

Since $\left|\left\{\pi \in S_{N} / \operatorname{rank}_{\pi}(e)<\operatorname{rank}_{\pi}(i)\right\}\right|=n ! / 2$, therefore the additional gain that $e$ gets due to $\alpha$ is $\Delta_{e}^{\mu}=\mu_{e}\left(v^{\alpha}\right)-\mu_{e}(v)=$ $(n ! / 2)(\alpha / n !)=(1 / 2) \alpha$, so

$$
\mu_{e}\left(v^{\alpha}\right)=\mu_{e}(v)+\frac{1}{2} \alpha
$$

Since $(1 / 2) \alpha+(1 / 2) \alpha=\alpha$, we deduce that, under the Shapley value, player $i$ transfers no fund to any fellow player.

So, half of each player's production is used to pay his salary, while the enterprise keeps half of the total production.

Proposition 9. Under our model of enterprise, the Shapley value is given by

$$
\begin{gathered}
\mu_{i}(v)=\frac{1}{2} q_{i}, \quad \forall i \in \widetilde{N}, \\
\mu_{e}(v)=\frac{1}{2} \sum_{i \in \widetilde{N}} q_{i} .
\end{gathered}
$$

Proof. With the notations in the proof of Proposition 8, remark that $v=T_{n}^{q_{n}}\left(\cdots\left(T_{2}^{q_{2}}\left(T_{1}^{q_{1}}\left(v_{0}\right)\right)\right)\right)$, apply $n$ iterations of $\left(\mathrm{R} 3^{\prime}\right)$ and (R4), and then conclude.

\subsection{Individual Shares under the Solidarity Value in Our Model} of Enterprise. Let us fix $j \in N \backslash\{i, e\}$. We need to compute the following:

$$
\begin{aligned}
\Delta_{i}^{\Psi}(\pi) & =\widehat{\Psi}_{i}^{v^{\alpha}}(\pi)-\widehat{\Psi}_{i}^{v}(\pi) \text { is what } i \text { keeps, } \\
\Delta_{e}^{\Psi}(\pi) & =\Psi_{e}^{v^{\alpha}}(\pi)-\Psi_{e}^{v}(\pi)(i \text { leaves to } e), \\
\Delta_{j}^{\Psi}(\pi) & =\Psi_{j}^{v^{\alpha}}(\pi)-\Psi_{j}^{v}(\pi) \text { is what } i \text { transfers to } j .
\end{aligned}
$$

For every pairwise distinct $u, v, r \in\{i, e, j\}$ denote

$L_{u v r}=\left\{\pi \in S_{N} / \operatorname{rank}_{\pi}(u)<\operatorname{rank}_{\pi}(v)<\operatorname{rank}_{\pi}(r)\right\}$.

The differences $\widehat{\Delta}_{k}^{\Psi}(\pi)=\widehat{\Psi}_{k}^{\alpha^{\alpha}}(\pi)-\widehat{\Psi}_{k}^{v}(\pi), k \in\{i, e, j\}$, are given by Table 4 .

We see from Table 4 that $\widehat{\Delta}_{j}^{\Psi}(\pi)$ does not vary with $j \neq i$ and that $\widehat{\Delta}_{e}^{\Psi}(\pi)=\widehat{\Delta}_{i}^{\Psi}(\pi)$. This implies that $\Delta_{j}^{\Psi}$, which is equal to $(1 /(n+1) !) \sum_{\pi \in S_{N}} \widehat{\Delta}_{j}^{\Psi}(\pi)$, does not vary with $j \neq i$, and that $\Delta_{e}^{\Psi}=\Delta_{i}^{\Psi}$. Let us first compute $\Delta_{j}^{\Psi}$ : we see from Table 4 that whenever $\pi \in L_{i e j} \cup L_{e i j}, \widehat{\Delta}_{j}^{\Psi}(\pi)=0$. For every $u v r \epsilon$ $\{i j e, e j i, j i e, j e i\}$ and $s \in\{3,4, \ldots, n+1\}$, denote

$$
L_{u v r}^{s}=\left\{\pi \in L_{u v r} / \operatorname{rank}_{\pi} r=s\right\} .
$$
by

If $\pi \in L_{u v r}^{s}, \widehat{\Delta}_{j}^{\Psi}(\pi)=\alpha / s$. Every $\pi \in L_{u v r}^{s}$ is characterized

(1) $u \in \pi_{\leq s-1}, v \in \pi_{\leq s-1}, \operatorname{rank}_{\pi} u<\operatorname{rank}_{\pi} v$,

(2) $\pi_{r}=s$.

Therefore,

$$
\begin{aligned}
\left|L_{u v r}^{s}\right| & =C_{s-1}^{2} \times((n+1-3)) ! \\
& =\frac{(s-2)(s-1)}{2}(n-2) !
\end{aligned}
$$

and we deduce, given Table 4, that

$$
\begin{aligned}
\Delta_{j}^{\Psi} & =\frac{1}{(n+1) !} \sum_{s=3}^{n+1} \sum_{u v r \in\{i j e, e j i, j i e, j e i\}}\left|L_{u v r}^{s}\right| \frac{\alpha}{s} \\
& =\frac{1}{(n+1) !} 4 \sum_{s=3}^{n+1}\left|L_{u v r}^{s}\right| \frac{\alpha}{s} \\
& =\frac{1}{(n+1) !} 4 \sum_{s=3}^{n+1} \frac{(s-2)(s-1)}{2}(n-2) ! \frac{\alpha}{s} \\
& =\frac{2}{(n-1) n(n+1)} \sum_{s=3}^{n+1} \frac{(s-2)(s-1)}{s} \alpha .
\end{aligned}
$$

For every $n \geq 2$, denote

$$
\Gamma(n)=\frac{2}{(n-1) n(n+1)} \sum_{s=3}^{n+1} \frac{(s-2)(s-1)}{s} .
$$

But since $(s-2)(s-1) / s=s+2 / s-3$, then $\sum_{s=3}^{n+1}((s-2)(s-$ $1) / s)=(n-2)(n-1) / 2+2\left(\sum_{s=3}^{n+1}(1 / s)\right)$. So, straightforward simplifications give the following expression of $\Gamma(n)$ which is useful for limits, although longer to write:

$$
\Gamma(n)=\frac{1}{n(n+1)}\left(n-2+\frac{4}{n-1}\left(\sum_{s=3}^{n+1} \frac{1}{s}\right)\right) .
$$

Then, we have $\Delta_{j}^{\Psi}=\Gamma(n) \alpha$, and $\Gamma(n)$ is the proportion of additional gain that player $j$ receives, due to $\alpha$. The total quantity of all transfers to the other $n-1$ players of $\widetilde{N}$ is 
TABLE 4

\begin{tabular}{|c|c|c|c|c|c|c|}
\hline$L_{u v r}$ & $L_{i e j}$ & $L_{e i j}$ & $L_{i j e}$ & $L_{e j i}$ & $L_{j i e}$ & $L_{j e i}$ \\
\hline$\widehat{\Delta}_{j}^{\Psi}(\pi)$ & 0 & 0 & $\alpha / \operatorname{rank}_{\pi}(e)$ & $\alpha / \operatorname{rank}_{\pi}(i)$ & $\alpha / \operatorname{rank}_{\pi}(e)$ & $\alpha / \operatorname{rank}_{\pi}(i)$ \\
\hline$\widehat{\Delta}_{e}^{\Psi}(\pi)$ & $\alpha / \operatorname{rank}_{\pi}(e)$ & $\alpha / \operatorname{rank}_{\pi}(i)$ & $\alpha / \operatorname{rank}_{\pi}(e)$ & $\alpha / \operatorname{rank}_{\pi}(i)$ & $\alpha / \operatorname{rank}_{\pi}(e)$ & $\alpha / \operatorname{rank}_{\pi}(i)$ \\
\hline$\widehat{\Delta}_{i}^{\Psi}(\pi)$ & $\alpha / \operatorname{rank}_{\pi}(e)$ & $\alpha / \operatorname{rank}_{\pi}(i)$ & $\alpha / \operatorname{rank}_{\pi}(e)$ & $\alpha / \operatorname{rank}_{\pi}(i)$ & $\alpha / \operatorname{rank}_{\pi}(e)$ & $\alpha / \operatorname{rank}_{\pi}(i)$ \\
\hline
\end{tabular}

therefore equal to $(n-1) \Gamma(n) \alpha$, and the amount left to $i$ and $e$ for equal sharing is $\alpha-(n-1) \Gamma(n) \alpha$. So, we have

(1) $\Delta_{i}^{\Psi}=\Delta_{e}^{\Psi}=((1-(n-1) \Gamma(n)) / 2) \alpha$,

(2) $\forall j \in N \backslash\{i, j\}, \Delta_{j}^{\Psi}=\Gamma(n) \alpha$.

We deduce that

$$
\begin{aligned}
& \Psi_{i}\left(v^{\alpha}\right)=\Psi_{i}(v)+\Delta_{i}^{\Psi}=\Psi_{i}(v)+\frac{1-(n-1) \Gamma(n)}{2} \alpha \\
& \Psi_{j}\left(v^{\alpha}\right)=\Psi_{j}(v)+\Delta_{j}^{\Psi}=\Psi_{j}(v)+\Gamma(n) \alpha \\
& \Psi_{e}\left(v^{\alpha}\right)=\Psi_{e}(v)+\frac{1-(n-1) \Gamma(n)}{2} \alpha .
\end{aligned}
$$

We therefore have the following result.

Proposition 10. Under our model of enterprise, the solidarity value is given by

$$
\begin{gathered}
\Psi_{i}(v)=\frac{1-(n-1) \Gamma(n)}{2} q_{i}+\Gamma(n) \sum_{j \in \widetilde{N} \backslash\{i\}} q_{j}, \\
\Psi_{e}(v)=\frac{1-(n-1) \Gamma(n)}{2} \sum_{j \in \widetilde{N}} q_{j},
\end{gathered}
$$

where $\Gamma(n)=\frac{2}{n(n+1)(n-1)} \sum_{s=3}^{n+1} \frac{(s-1)(s-2)}{s}$.

Proof. The proof is similar to that of Propositions 8 and 9. Iterate (F3) $n$ times and conclude.

5.3. Solidarity Flows in the Model of Enterprise. From the expression of $\Psi_{i}(v)$ that we have obtained in Proposition 10, we note that $\Psi_{i}(v)$ is an accumulation of funds from $n$ origins.

(1) The amount $((1-(n-1) \Gamma(n)) / 2) q_{i}$ is what $i$ keeps for himself while managing $q_{i}$. He transfers $\Gamma(n) q_{i}$, a constant amount to each of the other players $j \neq i$ (and therefore a total of $(n-1) \Gamma(n))$, and he transfers $((1-(n-1) \Gamma(n)) / 2) q_{i}$ to the enterprise.

(2) $\Gamma(n) \sum_{j \in \widetilde{N} \backslash\{i\}} q_{j}$ is a result of solidarity gifts from the other players in $\widetilde{N} \backslash\{i\}$, each $j \in \widetilde{N} \backslash\{i\}$ transferring to $i$ the amount $\Gamma(n) \sum_{j \in \widetilde{N} \backslash\{i\}} q_{j}$. The amount is proportional to $q_{j}$ but does not care whether or not the payee player $i$ is a needy. In Figure 1, we plot curves of the above encountered rates of transfer which only depends on the number of players.

There are so many player to player cross transfers. So, it is important to see in the outcome, and after all compensations,

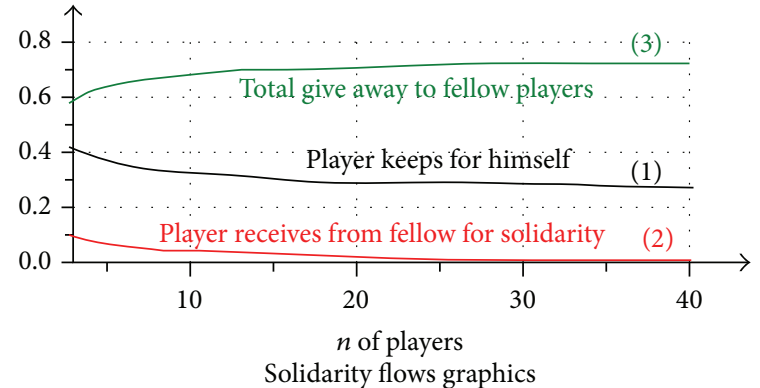

Plotting the rates of flows

(1) $1-(n-1) \Gamma(n) / 2$

(2) $\Gamma(n)$

(3) $(n-1) \Gamma(n)$

FIGURE 1

who benefits from the solidarity policy embodied in the solidarity value. Let $i \in N$. The Shapley value gives $\mu_{i}(v)=$ $(1 / 2) q_{i} \forall i \in \widetilde{N}$, and $\mu_{e}(v)=(1 / 2) \sum_{i \in \widetilde{N}} q$. We have

$$
\begin{aligned}
\Psi_{i}(v)-\mu_{i}(v) & =\frac{1-(n-1) \Gamma(n)}{2} q_{i}+\Gamma(n) \sum_{j \in \widetilde{N} \backslash\{i\}} q_{j}-\frac{1}{2} q_{i} \\
& =\frac{1}{2}\left[-q_{i}+\left(\frac{2}{|\widetilde{N}|} \sum_{j \in \widetilde{N}} q_{j}\right)\right](n+1) \Gamma(n), \\
\Psi_{e}(v)-\mu_{e}(v) & =\frac{1-(n-1) \Gamma(n)}{2} \sum_{j \in \widetilde{N}} q_{j}-\frac{1}{2} \sum_{i \in \widetilde{N}} q_{i} \\
& =\frac{-(n-1)}{2} \Gamma(n) \sum_{j \in \widetilde{N}} q_{j} .
\end{aligned}
$$

The number $\tau(n)=((n+1) / 2) \Gamma(n)$ appears as a factor in every player's solidarity tax calculation. Since $\Gamma(n)=(1 / n(n+$ 1)) $\left(n-2+(4 /(n-1))\left(\sum_{s=3}^{n+1}(1 / s)\right)\right)$, we have

$$
\tau(n)=\frac{1}{2}-\frac{1}{n}+\frac{2}{n(n-1)}\left(\sum_{s=3}^{n+1} \frac{1}{s}\right) .
$$

We plot the function $n \mapsto \tau(n)$ in Figure 2 .

From (37), we see that $b=(2 /|\widetilde{N}|) \sum_{j \in \widetilde{N}} q_{j}$ is the threshold of eligibility to solidarity help.

(1) If $q_{i}=b$, then $\Psi_{i}(v)=\mu_{i}(v)$; hence player $i$ receives no solidarity gift in the outcome.

(2) If $q_{i}<b$, then $\Psi_{i}(v)>\mu_{i}(v)$ so he receives some solidarity help which amounts to $\left(b-q_{i}\right) \tau(n)$. 


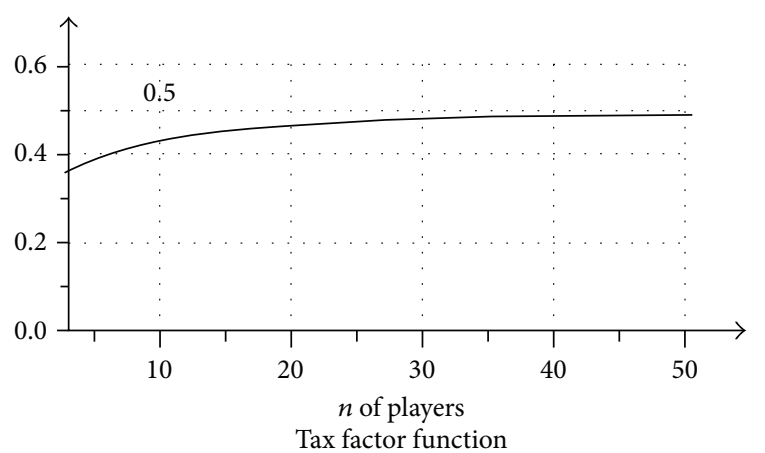

FIGURE 2

(3) If $q_{i}>b$, then $\Psi_{i}(v)<\mu_{i}$, so he gives away some funds which amount to $\left(q_{i}-b\right) \tau(n)$ for solidarity contribution.

The total assistance fund paid by the players excluding the enterprise is $T=\sum_{j \in \widetilde{N}} \Psi_{i}(v)-\mu_{i}(v)$, which is expressed by

$$
T=\tau(n) \sum_{j \in \widetilde{N}} q_{j}
$$

If $q_{j}, j \in N$, all satisfy $q_{j} \geq 0$, or more generally if $\sum_{j \in \widetilde{N}} q_{j} \geq 0$, we can see that the enterprise also pays part of the bill for solidarity assistance and receives no solidarity transfer. Its expenses amount to $(-(n-1) / 2) \Gamma(n) \sum_{j \in \widetilde{N}} q_{j}$.

Therefore, as for additive games, only a wealth player pays solidarity contribution in the outcome that is proportional to $q_{i}-b$, which is an indicator of his degree of wealth. The needy get solidarity help which is proportional to $b-q_{i}$ which may be regarded as a measure of the degree of poverty. What differs in the model of enterprise is that a larger number of players get help, since the thresholds are $b=(2 /|N|) \sum_{j \in N} q_{j}$ for the model of enterprise and $a=(1 /|N|) \sum_{j \in N} q_{j}$ for additive games.

\subsection{Solidarity Value as a Policy for Revenue Redistribution.} In a country, citizens pay tax while they earn an income. The government collects tax as funds for solving issues of common interest (building of roads, e.g.) or for social duties (such as ensuring low cost education and health care, or paying some solidarity revenue to the jobless people). The fulfillment of these duties may be regarded as a way of redistributing back the revenue collected to the citizens. In most cases, the collection of tax is done when and where transactions take place. If $i$ is a citizen, we regard $q_{i}$ as the income that $i$ produces if he is involved in a legal business with any group of partners. Of course, this is a very simplified model for a country with a very poorly structured economy. In the normal case, his productivity $q_{i}$ may also vary with his business partners. However, this example is a test to understand how the solidarity value works. The main challenge is what portion of an income should the state withhold as tax contribution? In this section, we want to consider the solidarity value as a basis for that redistribution.
Let us consider the citizens as the players and the state as the enterprise. Usually, $n$ is large in this case (e.g., 21 million for Cameroon). But we have

$$
\Psi_{i}(v)=\left[-q_{i}+\left(\frac{2}{|\widetilde{N}|} \sum_{j \in \widetilde{N}} q_{j}\right)\right] \tau(n)+\frac{1}{2} q_{i} .
$$

Since $\tau(n)=1 / 2-1 / n+(2 / n(n-1))\left(\sum_{s=3}^{n+1}(1 / s)\right)$, then we deduce that $\lim _{n \rightarrow \infty} \tau(n)=1 / 2$. We restrict ourselves to the class of games $(N, v)$ where $N$ is of any cardinality and the quota system $q=\left(q_{1}, q_{2}, \ldots, q_{n}, 0\right)$ is any $n+1$-uplet with the last component null, but where all $(N, v)$ in that class have the same average quota $b=(1 /|\widetilde{N}|) \sum_{j \in \widetilde{N}} q_{j}$ which is fixed; for example, let us say $b=1$. For each $(N, v)$ in that class and any $i \in N$, we have

$$
\Psi_{i}(v)=\left(-q_{i}+2 b\right) \tau(n)+\frac{1}{2} q_{i}
$$

The trouble here is that

$$
\lim _{n \rightarrow \infty}\left(\left(-q_{i}+2 b\right) \tau(n)+\frac{1}{2} q_{i}\right)=b .
$$

Take an individual $i$ and consider the subclass of all games $(N, v)$ such that $i \in N$ and $q_{i}=4$ for any such game. Then we will have $\Psi_{i}(v)=(-4+2 b) \tau(n)+2$. Then there exists $n_{0} \in \mathbb{N}$ such that $\left|\Psi_{i}(v)-b\right|<10^{-20}$, irrespective of the game $(N, v)$, provided that $|N|>n_{0}$.

So, in this context of a large value of $n$, the solidarity value will tend to allocate the average $q_{j}, j \in N$, to every citizen and therefore tend to empty the state coffer, since there will be nothing left to $e$. We conclude that the solidarity value will deny individual merit and empty the state coffers.

\section{Concluding Comments}

In a general TU game, a player will not, while paying his solidarity bill, reverse his social condition, if his privileged position is acknowledged by the desirability relation (see Theorem 5). For the two classes of games studied here, the needy actually receive help, and we have obtained the threshold of access to solidarity help in the outcome for the two classes of Sections 4 and 5. On how accurately solidarity actions target the players of poor condition, our analysis shows that player to player transfers of solidarity funds ignore the condition of the payee. Surprisingly and fortunately, it happens in the outcome after all compensations that only the needy get solidarity help which is proportional to their degree of poverty. About the amount of the bill of solidarity interventions, only wealthy people pay, each disbursing an amount which is proportional to his degree of wealth. Our last note points out some shortcomings of the solidarity value as a means for redistributing the state revenue: for large populations, it will ignore individual merits of citizens by distributing the national revenue in equal shares, and it will empty the state coffers.

We have obtained full answers to the questions who pays solidarity bills, for additive games and the model of enterprise. 
This study needs to be extended to more general TU games. The main challenge is the need of a suitable approach when it is not possible to fully describe a player $i$ using a quota $q_{i}$. Also, other variants of value for TU games related to sharing the join gains of cooperation, that have been introduced in the literature of TU game, equally need attention to investigate at least the various flows of solidarity funds and their fairness: see Chameni Nembua and Andjiga, (2008) [15], Chameni Nembua, (2012) [16], and Chameni Nembua and Themoi, (2013) [6].

\section{Disclosure}

This work was presented at the monthly seminar of Mathématiques Appliquées aux Sciences Sociales (MASS) at the Mathematics Department of the Faculty of Sciences of the University of Yaounde 1 in May 2014.

\section{Conflict of Interests}

The authors declare that there is no conflict of interests regarding the publication of this paper.

\section{Acknowledgments}

This work owes a significant improvement from an earlier version to suggestions from Professor Nicolas Gabriel ANDJIGA. The authors thank the participants, especially Dr. Moyouwou ISSOFA and Professor Bertrand TCHANTCHO, for very helpful comments. Also, they are indebted to three anonymous referees to whom they address special thanks.

\section{References}

[1] A. S. Nowak and T. Radzik, "A solidarity value for $n$-person transferable utility games," International Journal of Game Theory, vol. 23, no. 1, pp. 43-48, 1994.

[2] L. S. Shapley, "A value for n-person games," in Contributions to the Theory of Games, Volume II, pp. 307-317, Princeton University Press, Princeton, NJ, USA, 1953.

[3] A. C. Pigou, Wealth and Welfare, Macmillan, London, UK, 1912.

[4] H. Dalton, "The measurement of the inequality of incomes," The Economic Journal, vol. 30, no. 119, pp. 348-361, 1920.

[5] U. Ebert, "The decomposition of inequality reconsidered: weakly decomposable measures," Mathematical Social Sciences, vol. 60 , no. 2, pp. 94-103, 2010.

[6] C. Chameni Nembua and D. Themoi, "Ordinal equivalence of values and Pigou-Dalton transfers in TU-games," MPRA Paper 44895, University Library of Munich, Munich, Germany, 2013.

[7] J. R. Isbell, "A class of simple games," Duke Mathematical Journal, vol. 25, pp. 423-439, 1958.

[8] A. Taylor and W. Zwicker, "Weighted voting, multicameral representation, and power," Games and Economic Behavior, vol. 5, no. 1, pp. 170-181, 1993.

[9] A. D. Taylor and W. S. Zwicker, Simple Games: Desirability Relations, Trading, and Pseudoweightings, Princeton University Press, Princeton, NJ, USA, 1999.
[10] L. Diffo Lambo and J. Moulen, "Ordinal equivalence of power notions in voting games," Theory and Decision, vol. 53, no. 4, pp. 313-325, 2002.

[11] J. Friedman, L. McGrath, and C. Parker, "Achievable hierarchies in voting games," Theory and Decision, vol. 61, no. 4, pp. 305-318, 2006.

[12] F. Carreras and J. Freixas, "On ordinal equivalence of power measures given by regular semivalues," Mathematical Social Sciences, vol. 55, no. 2, pp. 221-234, 2008.

[13] B. Tchantcho, L. Diffo Lambo, R. Pongou, and B. Mbama Engoulou, "Voters' power in voting games with abstention: influence relation and ordinal equivalence of power theories," Games and Economic Behavior, vol. 64, no. 1, pp. 335-350, 2008.

[14] J. Freixas, "On ordinal equivalence of the Shapley and Banzhaf values for cooperative games," International Journal of Game Theory, vol. 39, no. 4, pp. 513-527, 2010.

[15] C. Chameni Nembua and N. Andjiga, "Linear, efficient and symmetric values for TU-games," Economics Bulletin, vol. 3, no. 71, pp. 1-10, 2008.

[16] C. Chameni Nembua, "Linear efficient and symmetric values for TU-games: sharing the joint gain of cooperation," Games and Economic Behavior, vol. 74, no. 1, pp. 431-433, 2012. 


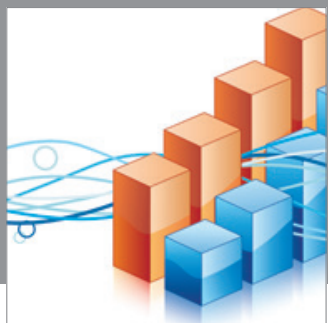

Advances in

Operations Research

mansans

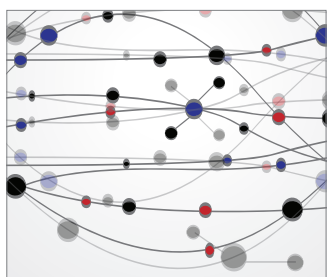

The Scientific World Journal
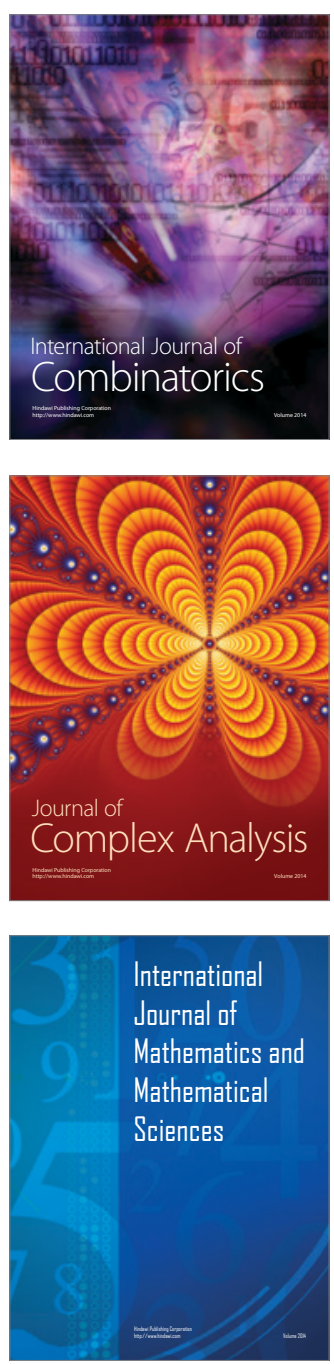
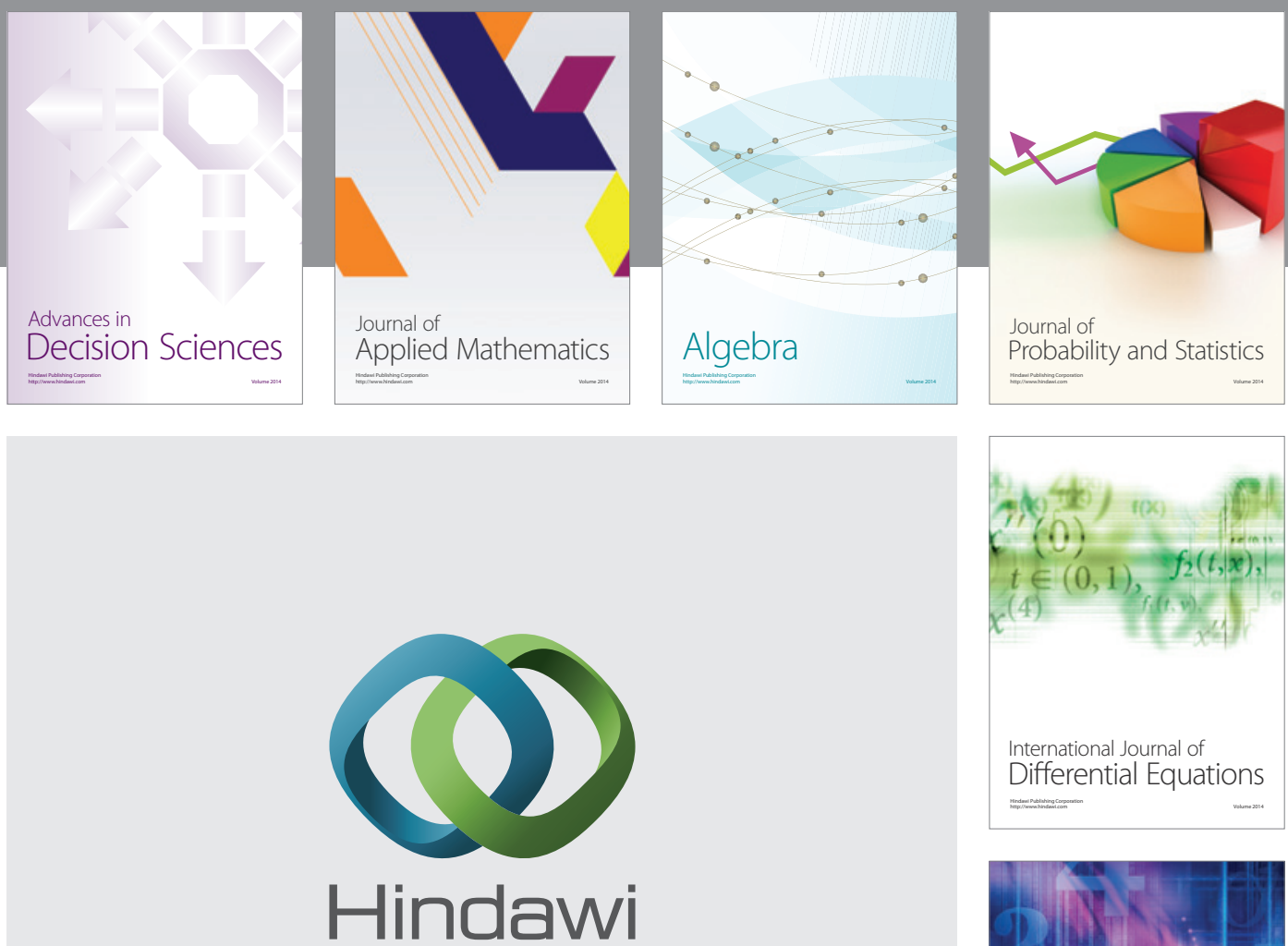

Submit your manuscripts at http://www.hindawi.com
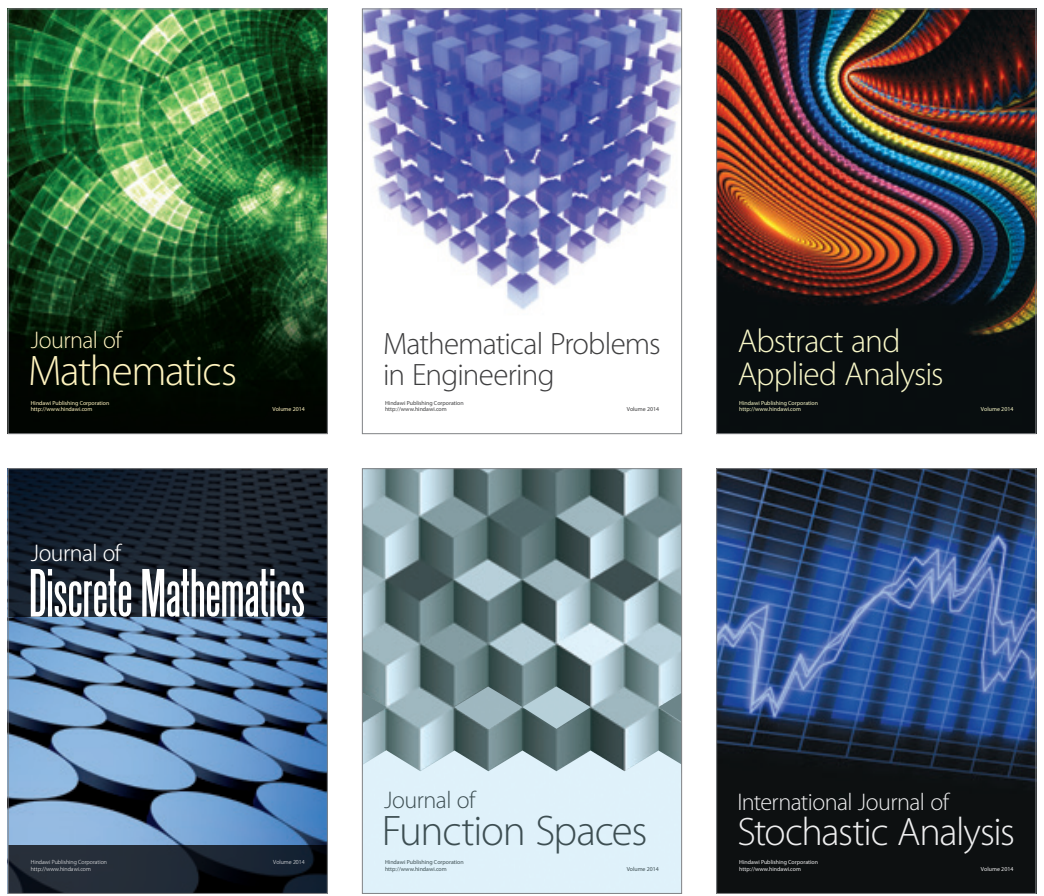

Journal of

Function Spaces

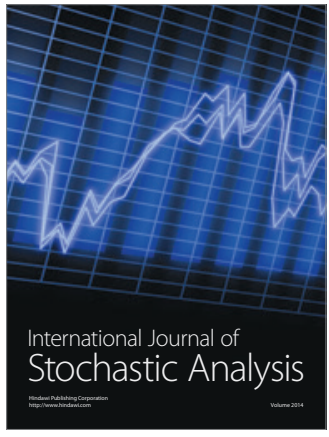

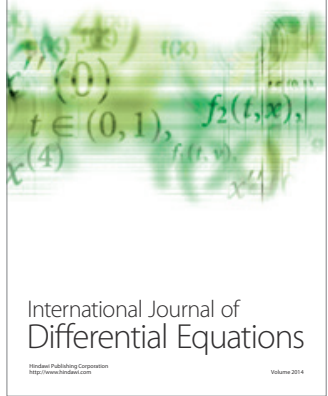
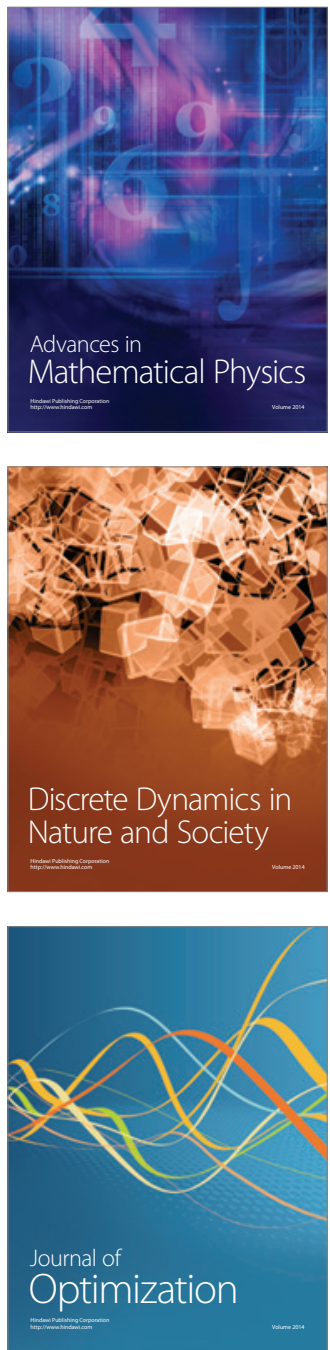